\title{
Analysis of the transcriptome of Panax notoginseng root uncovers putative triterpene saponin-biosynthetic genes and genetic markers
}

Hongmei Luo ${ }^{1 \dagger}$, Chao Sun ${ }^{1 \dagger}$, Yongzhen Sun ${ }^{1}$, Qiong $\mathrm{Wu}^{2}$, Ying Li ${ }^{1}$, Jingyuan Song ${ }^{1}$, Yunyun Niu', Xianglin Cheng ${ }^{3}$ , Hongxi Xu', Chuyuan Li ${ }^{5}$, Juyan Liư ${ }^{5}$, André Steinmetz ${ }^{6}$, Shilin Chen ${ }^{1 *}$

From BIOCOMP 2010. The 2010 International Conference on Bioinformatics and Computational Biology Las Vegas, NV, USA. 12-15 July 2010

\begin{abstract}
Background: Panax notoginseng (Burk) F.H. Chen is important medicinal plant of the Araliacease family. Triterpene saponins are the bioactive constituents in $P$. notoginseng. However, available genomic information regarding this plant is limited. Moreover, details of triterpene saponin biosynthesis in the Panax species are largely unknown.

Results: Using the 454 pyrosequencing technology, a one-quarter GS FLX titanium run resulted in 188,185 reads with an average length of 410 bases for $P$. notoginseng root. These reads were processed and assembled by 454 GS De Novo Assembler software into 30,852 unique sequences. A total of $70.2 \%$ of unique sequences were annotated by Basic Local Alignment Search Tool (BLAST) similarity searches against public sequence databases. The Kyoto Encyclopedia of Genes and Genomes (KEGG) assignment discovered 41 unique sequences representing 11 genes involved in triterpene saponin backbone biosynthesis in the 454-EST dataset. In particular, the transcript encoding dammarenediol synthase (DS), which is the first committed enzyme in the biosynthetic pathway of major triterpene saponins, is highly expressed in the root of four-year-old $P$. notoginseng. It is worth emphasizing that the candidate cytochrome P450 (Pn02132 and Pn00158) and UDP-glycosyltransferase (Pn00082) gene most likely to be involved in hydroxylation or glycosylation of aglycones for triterpene saponin biosynthesis were discovered from 174 cytochrome P450s and 242 glycosyltransferases by phylogenetic analysis, respectively. Putative transcription factors were detected in 906 unique sequences, including Myb, homeobox, WRKY, basic helix-loop-helix (bHLH), and other family proteins. Additionally, a total of 2,772 simple sequence repeat (SSR) were identified from 2,361 unique sequences, of which, di-nucleotide motifs were the most abundant motif.

Conclusion: This study is the first to present a large-scale EST dataset for $P$. notoginseng root acquired by nextgeneration sequencing (NGS) technology. The candidate genes involved in triterpene saponin biosynthesis, including the putative CYP450s and UGTs, were obtained in this study. Additionally, the identification of SSRs provided plenty of genetic makers for molecular breeding and genetics applications in this species. These data will provide information on gene discovery, transcriptional regulation and marker-assisted selection for $P$. notoginseng. The dataset establishes an important foundation for the study with the purpose of ensuring adequate drug resources for this species.
\end{abstract}

\footnotetext{
* Correspondence: slchen@implad.ac.cn

+ Contributed equally

${ }^{1}$ The Key Laboratory of Bioactive Substances and Resources Utilization of

Chinese Herbal Medicine, Ministry of Education, Institute of Medicinal Plant

Development, Chinese Academy of Medical Sciences \& Peking Union

Medical College, Beijing 100193, P. R. China

Full list of author information is available at the end of the article
} 


\section{Background}

Panax notoginseng (Burk) F.H. Chen is highly valued medicinal plant of the Araliaceae family [1]. P. notoginseng is distributed in the southwestern region of China, Burma and Nepal [2]. Presently, this species can only be found in cultivated forms [3]. In China, $P$. notoginseng is cultiviated commercially in Wenshan County, Yunnan province [4]. The roots of this plant, called notoginseng or sanchi, are commonly used as a hemostatic agent as well as a tonic to promote quality of life. In addition, the herb of sanqi possesses the bioactivities of antihypertensive, antithrombotic, anti-atherosclerotic and neuroprotective actions [3]. The ingredients detected in $P$. notoginseng include triterpene saponins, non-protein amino acids, polyacetylenes, phytosterols, flavonoids, and polysaccharides, many of which have pharmacological activities and are useful in the treatment of some diseases [2]. Among these compounds, triterpene saponins, a group of ginsenosides, are considered to be the principal bioactive components responsible for the pharmacological features [5-7]. Approximately 60 triterpene saponins have been isolated from $P$. notoginseng including ginsenosides, notoginsenosides, and gypenosides [2]. The major ginsenosides are the dammarane glycosides, and the ginsenoside Rg1, Rb1, Rd, and notoginsenoside $\mathrm{R} 1$ are considered as the major constituents found in the $P$. notoginseng root [8]. All the dammarane saponins have been classified as two groups: the protopanaxadiols group and the protopanaxatriols group [2]. The oleanane-type saponin, Ro, which exists in Asian ginseng (Panax ginseng) and American ginseng (Panax quinquefolius), have not been found in $P$. notoginseng based on the evidence from phytochemical studies [2].

Triterpene saponins are synthesized via the mevalonic acid (MVA) pathway [9], which is ubiquitous in plants and provides the precursor 2,3-oxidosqualene for terpenoid biosynthesis. The cyclization of 2,3-oxidosqalene by oxidosqualene cyclase (OSC) combined with the following modifications on the triterpene skeletons including hydroxylation and glycosidation leads to the production of various ginsenosides (Figure 1). The OSC genes including dammarenediol synthase $(D S), \beta$-amyrin $(\beta$ $A S)$, lupeol synthase $(L S)$ and cycloartenol synthase $(C A S)$ have been isolated in plants [10-13]. The characterization of DS, the first key enzyme committed in the biosynthesis of dammarane-type saponins, was profoundly advanced the studies on triterpene saponin biosynthesis in $P$. ginseng $[10,14]$. However, little is known about the molecular mechanism of the biosynthetic pathway downstream of cyclization involved in ginsenoside biosynthesis (Figure 1). Some specific cytochrome P450-dependent monoxygenases (CYP450s) and UDPglycosyltransferases (UGTs), which might be existed in
Panax plants, are proposed to catalyze the conversion of dammarenediol-II or $\beta$-amyrin to various ginsenosides and the modification on ginsenosides. In our previous study, one candidate CYP450 and four candidate UGTs most likely to be involved in ginsenoside biosynthesis have been selected from $P$. quinquefolius [15]. These candidate genes were screened from the 454-EST dataset of $P$. quinquefolius root based on the analysis of tissue-specific expression pattern and methyl jasmonate (MeJA) induction [15].

Despite its pharmacological importance, the transcriptomic and genomic data of $P$. notoginseng are very limited and only 95 ESTs are available in the National Center for Biotechnology Information (NCBI) database. The limited transcriptomic data hinder the study of triterpene saponin biosynthetic mechanisms in P. notoginseng. Expressed sequence tag (EST) analysis is a useful tool for the purposes of gene discovery especially in non-model plants for which no reference genome sequences are available [16]. ESTs represent the expressed portion of a genome $[17,18]$ and can be used to characterize patterns of gene expression in special tissues [19]. The discovery and prediction of genes involved in triterpene saponin and other secondary metabolite biosynthesis was performed based on EST analysis $[15,20,21]$. The triterpene carboxylic acid glucosyltransferase was characterized by mining ESTs from the developing seeds of Saponaria vaccaria [22]. The licorice-amyrin 11-oxidase gene, which plays a key role in the biosynthesis of the triterpene sweetener glycyrrhizin, was identified from the ESTs generated from the stolons of Glycyrrhiza uralensis [23]. In addition, ESTs are a rich source of gene-derived molecular markers (e. g. simple sequence repeat, SSR) which will be used for germplasm breeding or physical mapping [24]. The next-generation sequencing (NGS) technologies improve sequencing depth and render large-scale EST projects more feasible [25-27].

Herein we present the results of the study designed to characterize the transcriptome of $P$. notoginseng root using NGS technology based on 454 GS FLX Titanium platform. Our ultimate goal is to discover the candidate genes that encode enzymes in the triterpene saponin biosynthetic pathway and provide an overview of transcriptome, as well as produce molecular markers of EST-SSRs for facilitation the marker-assisted breeding of this species.

\section{Results and discussion}

\section{Transcriptome sequencing and sequence assembly}

A one-quarter 454 GS FLX Titanium run representing the cDNA library of 4-year old $P$. notoginseng root produced 188,185 reads with an average length of $410 \mathrm{bp}$ 


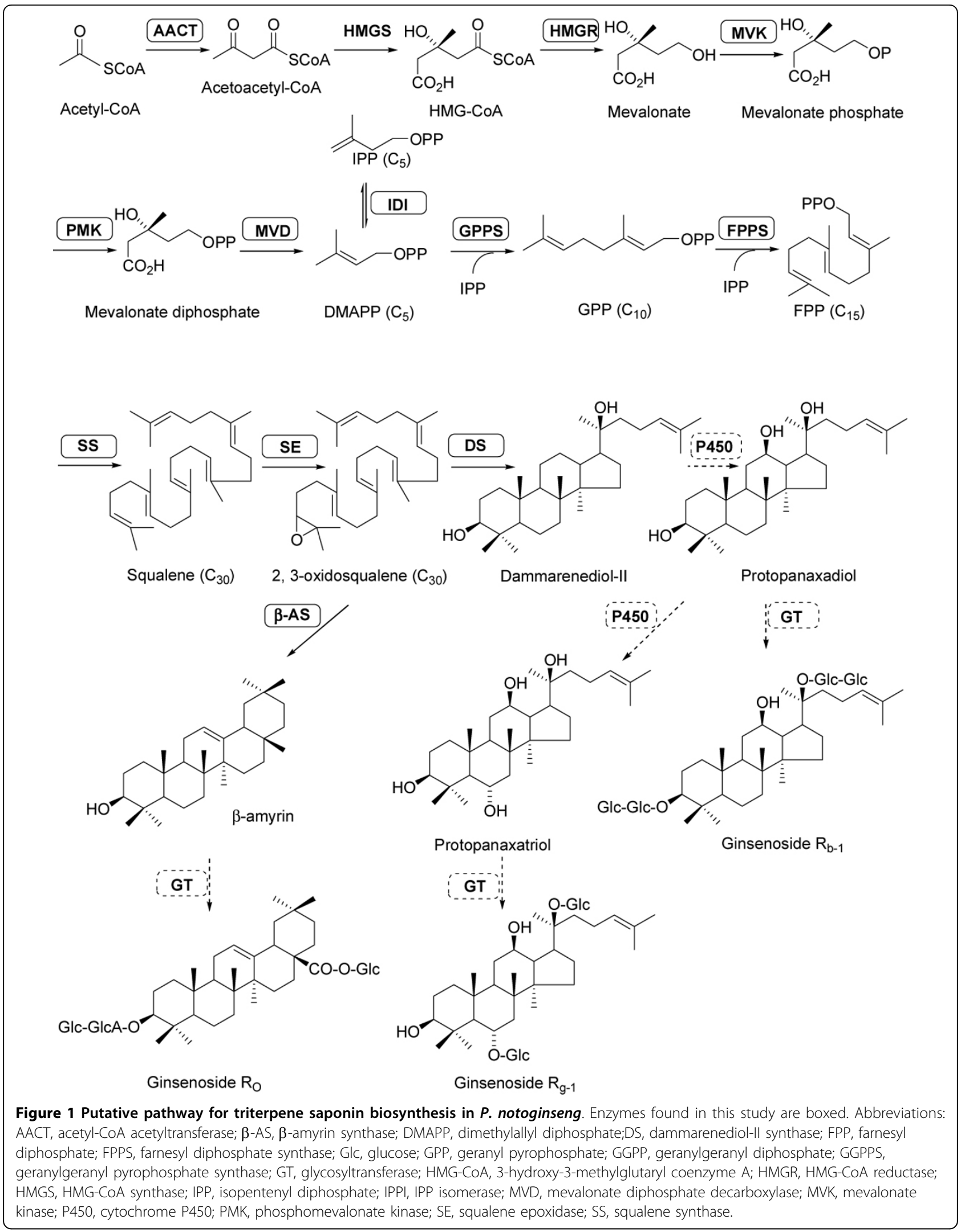


(Table 1). After trimming adaptor sequences and removing those reads shorter than $50 \mathrm{bp}$, a total of 184,785 reads were assembled into 30,852 unique sequences including 14,005 contigs and 16,847 singletons (Table 1). The lengths of contigs ranged from 95 to $10,423 \mathrm{bp}$ with average size of $581 \mathrm{bp}$ (Table 1). The size distributions for these reads and contigs are shown in Figure 2. Singletons, which represent unique transcripts expressed at low levels in the samples and with only one read, exhibited an average length of $343 \mathrm{bp}$ in $P$. notoginseng 454-EST dataset (Table 1). The length of these unique sequences was sufficient to enable annotations with high accuracy [28]. An overview of 454 sequencing and assembly for $P$. notoginseng was summarized in Table 1 .

\section{Unique sequence annotation and highly expressed transcript analyses}

The annotation for $P$. notoginseng unique sequences was based on sequence similarity searches against public databases. These databases included SwissProt, KEGG, The Arabidopsis Information Resource (TAIR), NCBI non-redundant protein $(\mathrm{Nr})$, and NCBI non-redundant nucleotide $(\mathrm{Nt})$ database. The number and percentage of the annotated unique sequences were summarized in Additional file 1. In total, 21,672 (70.2\%) P. notoginseng unique sequences were annotated and the remaining (29.8\%) unique sequences had no match to any sequences in the public databases (Table 1). The annotation rate for $P$. notoginseng unique sequences is similar to that of $P$. quinquefolius root [15] and $P$. ginseng root [29] transcriptomes. The comparisons of the 30,852 $P$. notoginseng unique sequences with all the $P$. quinquefolius [15] and $P$. ginseng [29] unique sequences derived from 454 sequencing using BLAST search ( $E$-value <=

Table 1 Summary of 454 sequencing and assembly for $P$. notoginseng

\begin{tabular}{lll}
\hline & No. of sequences & No. of bases \\
\hline HQ reads & 188,185 & $77,079,504$ \\
Average HQ read length & $410 \pm 138 \mathrm{bp}$ & \\
Reads used in assembly & 184,785 & $75,152,969$ \\
\hline Reads assembled as contigs & 164,855 & $65,067,207$ \\
Number of contigs & 14,005 & $8,131,261$ \\
Average length of contigs & $581 \pm 404 \mathrm{bp}$ & \\
Range of contig lengths & $95-10,423 \mathrm{bp}$ & \\
Contigs above 200 bp & 12,809 & $7,956,026$ \\
\hline Number of singletons & 16,847 & $5,775,116$ \\
Average length of singletons & $343 \pm 166 \mathrm{bp}$ & \\
Range of singleton lengths & $50-728 \mathrm{bp}$ & \\
Singletons above 200 bp & 12,365 & $5,310,178$ \\
\hline Number of unique sequences & & \\
Unique sequences above 200 bases $^{\mathrm{a}}$ & 25,174 & \\
\hline
\end{tabular}

${ }^{a}$ The total number of contigs and singletons.

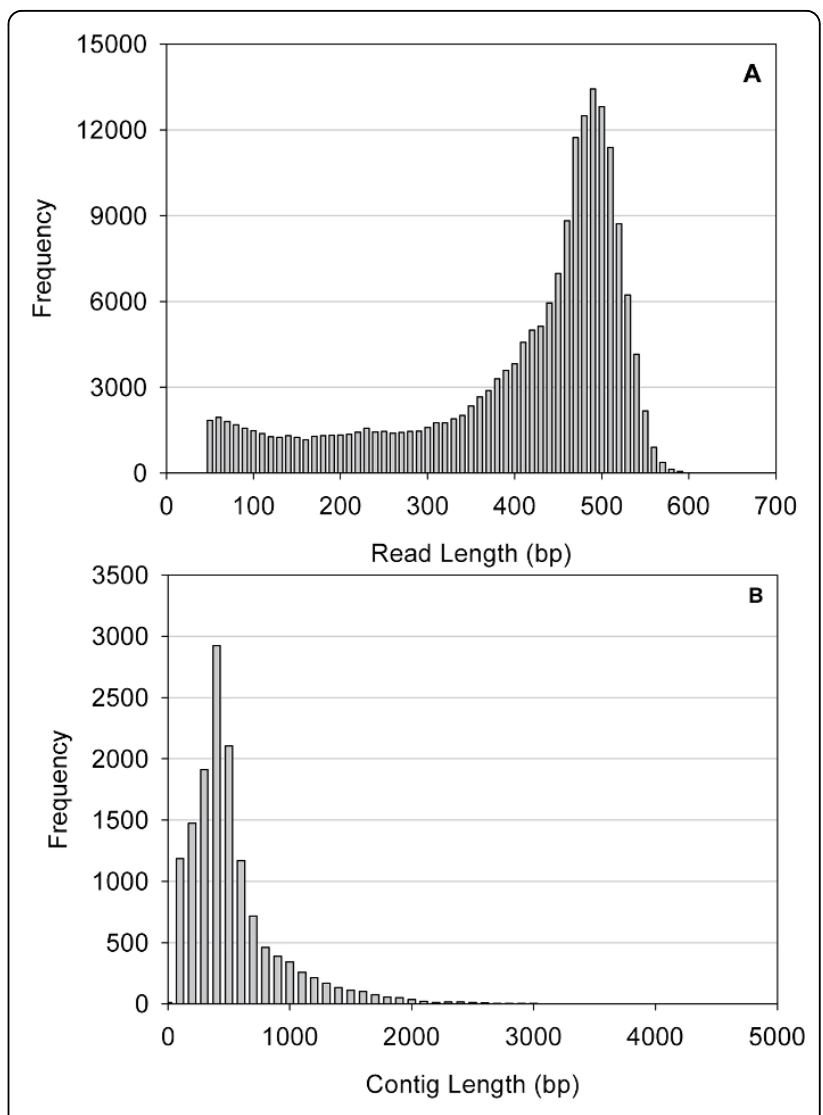

Figure 2 Length distributions of reads and contigs of $P$. notoginseng. A. Size distribution of 454 sequencing reads of $P$. notoginseng after removal of adaptor sequences. B. Length distribution of contigs (assembled sequences) in P. notoginseng 454EST dataset.

1e-10) were performed. The result of the comparison between $P$. notoginseng and $P$. quinquefolius [15] unique sequences showed that $19,226 P$. notoginseng unique sequences had sequence similarity to $P$. quinquefolius unique sequences, and the remained 11,626 unique sequences were the $P$. notoginseng special transcripts. Similarly, a total of $19,479 P$. notoginseng unique sequences had sequence similarity to $P$. ginseng unique sequences [29], and the rest 11,373 unique sequences were the $P$. notoginseng special transcripts.

The abundance of a transcript in a cDNA library from specific tissues/organs generally corresponds to its expression level in the original biological sample, which can indicate the ongoing biological processes [30]. The top 20 most frequent unique transcripts in the cDNA library of the 4-year old $P$. notoginseng root were shown in Table 2. The most abundant $P$. notoginseng transcript (with 3,358 reads) had no hit to any sequences of the public databases. The transcripts encoding enzymes associated with sugar and energy metabolism were highly expressed in this cDNA library, including 1,4- 
Table 2 The 20 most abundant transcripts in $P$. notoginseng root

\begin{tabular}{lllll}
\hline Rank & Contig & Gene ID & Gene name & No. of reads \\
\hline 1 & contig14005 & No hit & Reticuline oxidase -like protein & 3,358 \\
2 & contig13784 & sp|Q9SVG4| & P. ginseng-specific abundant protein 3 & 2,043 \\
3 & contig13829 & gb|AAX40471.1| & P. ginseng-specific abundant protein 3 & 1,949 \\
4 & contig13823 & gb|AY829463.1| & 1,4-alpha-glucan- branching enzyme & 1,748 \\
5 & contig00208 & sp|P30924| & Acetyl-CoA acetyltransferase & 1,301 \\
6 & contig00313 & dbj|BAF98277.1| & Heat shock protein, putative & 1,129 \\
7 & contig00270 & gb|EEF30081.1| & (S)-reticuline oxidase-like protein & 1,048 \\
8 & contig00110 & dbj|BAB68539.1| & P. ginseng dammarenediol-Il synthase & 1,018 \\
9 & contig13785 & dbj|BAF33291.1| & Delta12-fatty acid acetylenase & 1,009 \\
10 & contig13417 & gb|AAO38031.1| & P. ginseng squalene epoxidase & 865 \\
11 & contig00111 & gb|ACJ24907.2| & & 778 \\
12 & contig00323 & No hit & P. ginseng clone PG6L-4 & 747 \\
13 & contig13999 & gb|DQ384527.1| & Heat shock protein, putative & 742 \\
14 & contig00310 & gb|EEF49052.1| & Heat shock protein 70-3 & 711 \\
15 & contig00282 & gb|AAR17080.1| & Sucrose synthase isoform 1 & 616 \\
16 & contig00123 & sp|P49035| & Tonoplast intrinsic protein & 538 \\
17 & contig13552 & gb|ABB29477.1| & Formate dehydrogenase & 537 \\
18 & contig00095 & sp|Q07511| & Hypothetical protein & 527 \\
20 & contig00665 & ref|YP_588403.1| & Heat shock protein & 511 \\
\hline
\end{tabular}

alpha-glucan-branching enzyme protein, sucrose synthase isoform, and formate dehydrogenase (Table 2). The abundance of these transcripts was consistent with the fact that starch is the major constituent in the $P$. notoginseng root. It is noteworthy that three transcripts encoding the key enzymes of Acetyl-CoA acetyltransferase (AACT), squalene epoxidase (SE) and dammarenediol synthase (DS) were also abundant in $P$. notoginseng root (Table 2). These enzymes play important roles in triterpene saponin biosynthesis, particularly for DS, which is the first committed enzyme in the biosynthetic pathway of major triterpene saponins. In comparison, $D S$ was more abundant in $P$. notoginseng root than in $P$. quinquefolius [15] and P. ginseng root [29]. The high expression levels of these transcripts encoding DS indicated that triterpene saponin biosynthesis is active in the growth stage of 4-year old of this species. Therefore, the genes (e.g. some specific CYP450s and UGTs) involved in the downstream of triterpene saponin biosynthetic pathway are likely to be abundant in this 454EST dataset. In addition, several transcripts encoding heat shock proteins, which have the functions in the abiotic stress response in plants [31], were also expressed highly in P. notoginseng root (Table 2).

\section{GO analysis and KEGG assignment}

The GO annotation describes gene products according to their associated molecular functions, cellular components, and biological processes, illustrating the broad overview of the groups of genes cataloged in transcriptome [32]. A total of 18,689 P. notoginseng unique sequences were assigned with GO terms based on sequence similarity to proteins in TAIR database (Figure 3). In cellular component group, the unique sequences related to the Golgi apparatus, mitochondria, ribosome, and cell wall were well-represented categories (Figure 3A). The transcripts belonging to the major subgroups of molecular function category included protein binding, kinase activity, and receptor binding or activity (Figure 3B). The best-represented groups of biological processes were response to abiotic or biotic stimuli, protein metabolism, cell organization and biogenesis (Figure 3C). These GO annotations provide a comprehensive information on transcript functions of $P$. notoginseng.

The KEGG assignments provide an alternative functional annotation of genes associated biochemical pathways with their corresponding enzyme commission (EC) numbers [33]. 16,300 P. notoginseng unique sequences were assigned to KEGG annotations based on sequence similarity searches against the KEGG database. A total of 3,862 unique sequences among the 16,300 annotated sequences were assigned to biochemical pathways (Additional file 2). The remaining $(12,438)$ unique sequences were not assigned to any known biochemical pathway (Additional file 2). In detail, a total of 1,349 unique sequences were assigned to the metabolic pathways, including the well-represented pathways of amino acid metabolism, carbohydrate metabolism, lipid metabolism, energy metabolism, and the biosynthesis of secondary metabolites (Additional file 2). The number of 


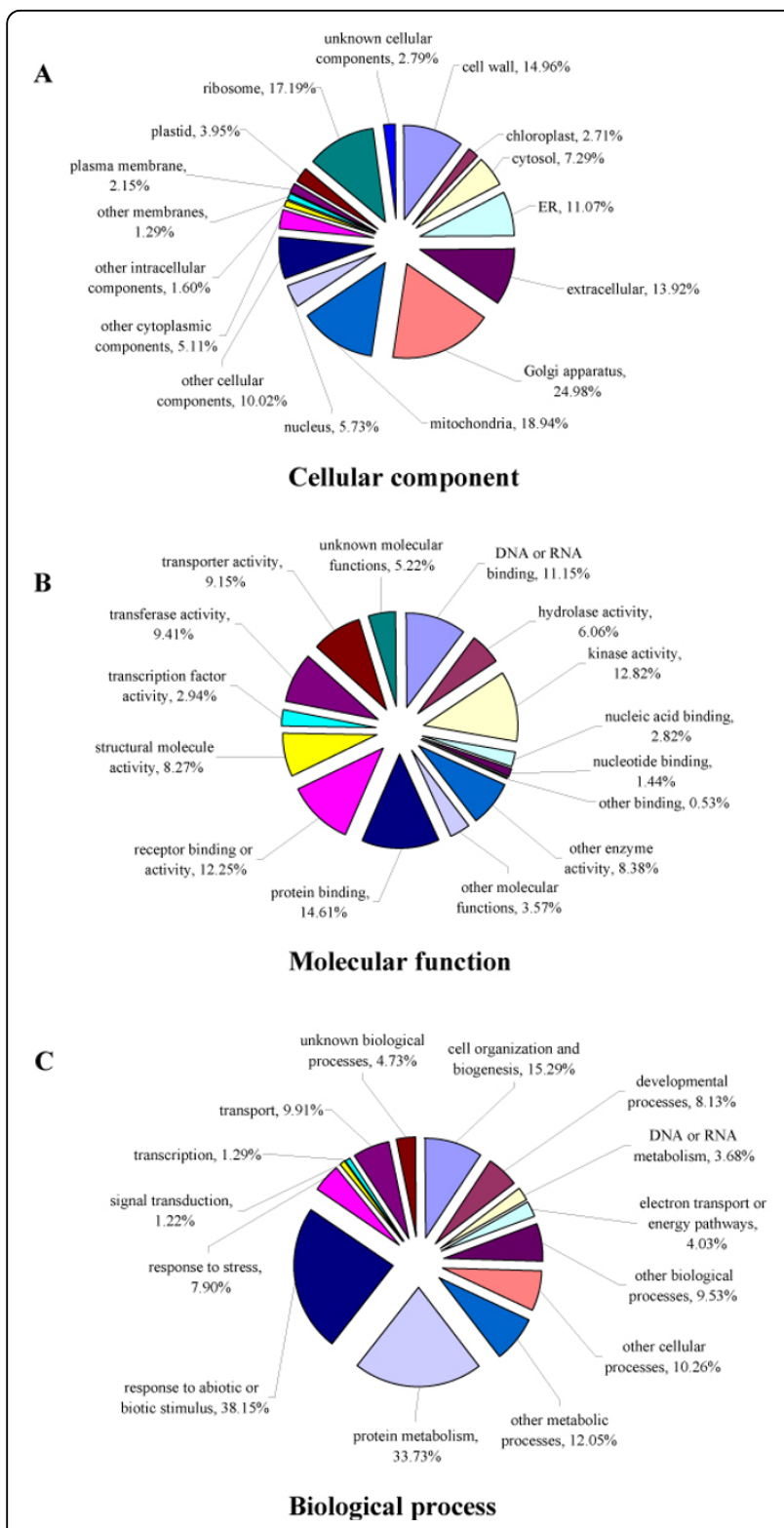

Figure $3 \mathrm{GO}$ analysis of $P$. notoginseng unique sequences based on cellular component (Figure $3 \mathrm{~A}$ ), molecular function (Figure 3B), and biological process (Figure 3C)

unique sequences involved in the biosynthesis of secondary metabolites was shown in Additional file 3. In particular, the transcripts encoding most of all the known enzymes (except the HMG-CoA synthase, HMGS) involved in the triterpene saponin biosynthesis were discovered in this 454-EST dataset, including acetyl-CoA acetyltransferase (AACT), HMG-CoA reductase (HMGR), mevalonate kinase (MVK), phosphomevalonate kinase (PMK), mevalonate diphosphate decarboxylase (MVD), IPP isomerase (IPPI), geranylgeranyl pyrophosphate synthase (GGPPS), farnesyl diphosphate synthase (FPPS), squalene synthase (SS), squalene epoxidase (SE), and dammarenediol-II synthase (DS) (Figure 1 andTable 3). Pathways related to genetic information processing, protein families, and cellular processes were also well represented by $P$. notoginseng unique sequences (Additional file 2).

\section{SSR detection}

Simple sequence repeats (SSRs) are the most feasible genetic markers for plant breeding and genetic applications [34]. A total of 2,772 putative SSR motifs were identified from 2,361 P. notoginseng unique sequences with $8.98 \%(2,772 / 30,852)$ frequency (Additional file 4). The frequency of SSRs identified among $P$. notoginseng 454-ESTs was similar to that of some dicotyledonous species [35]. These motifs included di-, tri-, tetra-, penta- and hexa-nucleotides with the lengths ranging from 2 to $6 \mathrm{bp}$. Among the SSR-containing unique sequences, the majority ( $85.51 \%)$ had a single SSR motif in every sequence.

The di-nucleotide motifs were the most abundant in 454-ESTs, which is similar to results obtained from other plants [36]. The occurrence of di- and tri-nucleotide SSR motifs and the number of repeats were presented in Table 4 . With a frequency of over sixty percent $(67.53 \%, 1,872 / 2,772)$, di-nucleotides were about two times more abundant than tri-nucleotides $(25 \%$, $693 / 2,772)$, followed by tetra-nucleotides $(3.79 \%, 105 /$ $2,772)$ and penta- and hexa-nucleotides (3.68\%, 102/ 2,772). Among the di-nucleotide repeat classes, AG/GA/ $\mathrm{CT} / \mathrm{TC}(57.3 \%)$ was the most frequent dimer motif. Other frequent dimer motifs included AT/TA and AC/ CA/GT/TG (Table 4). The CG repeats were very infrequent in the plant $(0.1 \%)$, which is consistent with previous observations [36-38]. Among the tri-nucleotide repeats, AAG/GAA/AGA/CTT/TTC/TCT was the largest repeat class followed by ATC/CAT/TCA/GAT/ ATG/TGA and AGC/CAG/GCA/TGC/CTG/GCT (Table 4). Our findings indicated that unique sequences containing SSR markers were indeed abundant in $P$. notoginseng. In particular, several SSR motifs were linked with the unique sequences encoding enzymes (e. g. AACT, HMGR, SE, SS, DS) involved in triterpene saponin biosynthesis (Additional file 5). These unique sequence-derived markers generated in this study represent a valuable genetic resource for future studies of this species as well as related Panax species.

\section{Discovery of transcripts encoding putative transcription factors in $P$. notoginseng}

Transcription factors, the sequence-specific DNA-binding proteins, play important roles in the regulation of gene expression in response to developmental programs and environmental changes in plants [39]. Based on the searches of automated predictions using Inter-Pro, a 
Table 3 Genes involved in triterpene saponin biosynthesis in P. notoginseng 454-EST dataset

\begin{tabular}{|c|c|c|}
\hline Gene name & EC & Unique sequences \\
\hline Acetyl-CoA acetyltransferase, $A A C T$ & 2.3.1.9 & $\begin{array}{l}\text { contig00313|contig11368|FW1NBNE02C59HZ| } \\
\text { FW1NBNE02DS7UV|FW1NBNE02EHI1C }\end{array}$ \\
\hline $\begin{array}{l}\text { HMG-CoA reductase, } \\
\text { HMGR }\end{array}$ & 1.1.1.34 & $\begin{array}{l}\text { contig00303|contig01 157|contig13725|contig13678| } \\
\text { contig00303|contig13324|FW1NBNE02DDW8P| } \\
\text { FW1NBNE02DH5GW }\end{array}$ \\
\hline Mevalonate kinase, MVK & 2.7.1.36 & contig06070 \\
\hline Phosphomevalonate kinase,PMK & 2.7.4.2 & FW1NBNE02DP41Y \\
\hline $\begin{array}{l}\text { Mevalonate diphosphate } \\
\text { decarboxylase, MVD }\end{array}$ & 4.1.1.33 & contig00126 \\
\hline IPP isomerase, IPPI & 5.3.3.2 & contig02936|contig02937|contig06726|contig06727 \\
\hline $\begin{array}{l}\text { Geranylgeranyl pyrophosphate } \\
\text { synthase, GGPPS }\end{array}$ & 2.5.1.29 & contig07732 \\
\hline $\begin{array}{l}\text { Farnesyl diphosphate } \\
\text { synthase, FPPS }\end{array}$ & 2.5.1.10 & contig02597|contig10067 \\
\hline Squalene synthase, SS & 2.5.1.21 & contig04447|contig07285 \\
\hline Squalene epoxidase, SE & 1.14.99.7 & $\begin{array}{l}\text { contig03811|contig13936|contig09188|contig00132| } \\
\text { contig00111|FW1NBNE02DZW52|FW1NBNE02C3G4P|FW1NBNE02C1AS6 }\end{array}$ \\
\hline Dammarenediol-II synthase, DS & & $\begin{array}{l}\text { contig13785|FW1 NBNE02EEIGL|FW1NBNE02DNRCV| } \\
\text { FW1NBNE02DNEAO|FW1NBNE02DIULM|FW1NBNE02DEHXE|FW1NBNE02DB02T| FW1NBNE02D0GUT }\end{array}$ \\
\hline
\end{tabular}

total of 906 P. notoginseng unique sequences representing putative homologs belonging to different transcription factor (TF) families (Additional file 6), covering the ARF, AUX/IAA, B3, MYB, basic Helix-Loop-Helix (bHLH), bZIP, Homeobox, Homeodomain-like/related, pathogenesis-related/ERF, WRKY and Zinc finger family proteins (Additional file 7). Many protein members of the MYB, bZIP and WRKY transcription factors have been implicated in the regulation of stress responses [39]. The most abundant TF family in P. notoginseng

Table 4 Summary of di- and tri-nucleotide repeats in $P$

\begin{tabular}{ll}
\hline Repeat composition & $\begin{array}{l}\text { No. of unique sequences } \\
\text { (relative percentage) }\end{array}$ \\
\hline Dinucleotide & $167(8.9 \%)$ \\
AC/CA/GT/TG & $1073(57.3 \%)$ \\
AG/GA/CT/TC & $630(33.7 \%)$ \\
AT/TA & $2(0.1 \%)$ \\
CG/GC & $\mathbf{1 8 7 2 ( 1 0 0 \% )}$ \\
Total of dinucleotide & \\
\hline Trinucleotide & $35(5.1 \%)$ \\
AAC/CAA/ACA/GTT/TTG/TGT & $152(21.9 \%)$ \\
AAG/GAA/AGA/CTT/TTC/TCT & $73(10.5 \%)$ \\
AAT/TAA/ATA/ATT/TTA/TAT & $62(8.9 \%)$ \\
ACC/CAC/CCA/GGT/GTG/TGG & $15(2.2 \%)$ \\
ACG/CGAGAC/CGT/GTC/TCG & $28(4 \%)$ \\
ACT/CTA/TAC/AGT/TAG/GTA & $116(16.7 \%)$ \\
AGC/CAG/GCA/TGC/CTG/GCT & $74(10.7 \%)$ \\
AGG/GGA/GAG/TCC/CTC/CCT & $113(16.3 \%)$ \\
ATC/CAT/TCA/GAT/ATG/TGA & $25(3.6 \%)$ \\
CCG/CGC/GCC/GGC/GCG/CGG & $\mathbf{6 9 3}(\mathbf{1 0 0 \% )}$ \\
Total of trinucleotide &
\end{tabular}

454-EST dataset was the MYB family proteins characterized by DNA-binding domains. In Arabidopsis, the MYB family, comprising 163 genes, is also one of the largest transcription factor families [40]. The Homeobox proteins were another set of highly expressed transcription factors in $P$. notoginseng. Homeobox genes regulate various developmental aspects in plants, such as the regulation of stem cell specification and organogenesis [41]. The high expression level of MYB and Homeobox proteins in $P$. notoginseng may be linked to response to specific habitats and developmental regulation. Given that the functions of TFs vary in plants, the putative functions of these transcription factors potentially involved in environmental responses and/or developmental regulation in $P$. notoginseng will be characterized in a future study. It is noteworthy that the discovery of these candidate TFs in our 454-EST dataset may provide useful information for future research.

\section{Candidate genes encoding enzymes involved in the biosynthesis of triterpene saponins}

\section{Discovery of the transcripts encoding the known enzymes} invioved in triterpene saponin biosynthesis

Most of all the known enzymes involved in MVA pathway for triterpene saponin biosynthesis were discovered in P. notoginseng 454-EST dataset (Table 3). As shown in Figure 1, the oxidosqualene is a precursor in the biosynthesis of triterpenoids in higher plants [9]. The cyclization of 2,3-oxidosqualene, catalyzed by OSCs (e.g. DS or AS), is the rate-limited step for triterpene saponin biosynthesis (Figure 1). After the cyclization, the hydroxylation and glycosidation, which are catalyzed by CYP450s and UGTs in turn, play important roles in the 
production of various triterpene saponins (Figure 1). Dammarane-type saponins are major saponins in $P$. notoginseng root. DS participates in the cyclization of 2,3-oxidosqualene to form the dammarane skeletons in $P$. ginseng $[10,14]$. It is noteworthy that the transcript for the full-length $P$. notoginseng $D S$ (containing 1,018 reads) was found in the cDNA library. The alignment of amino acid sequences of DS from $P$. ginseng, $P$. notoginseng, and $P$. quinquefolius was shown in Figure 4. DS sequences of $P$. quinquefolium and $P$. notoginseng were

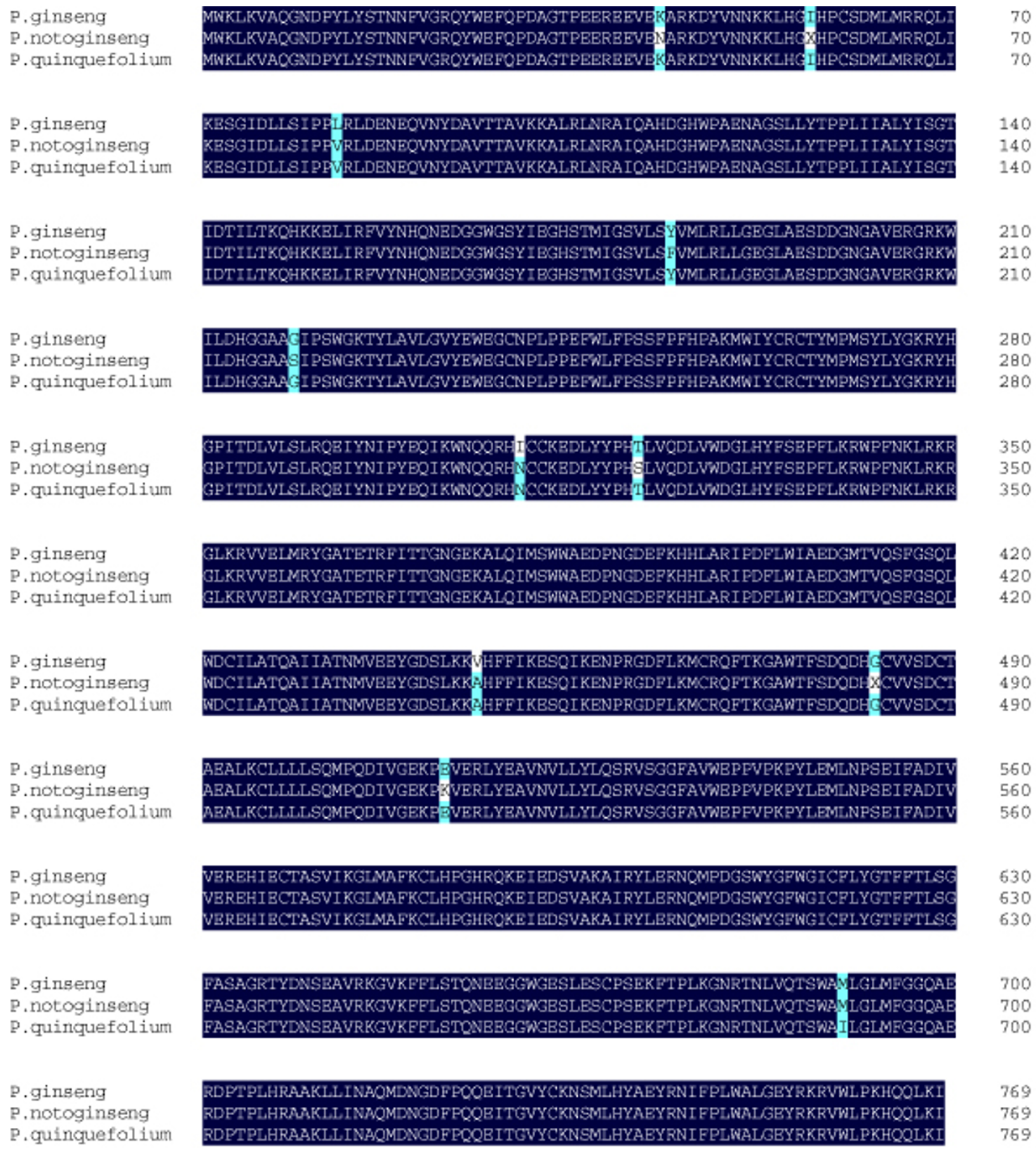

P.ginseng

P. notoginseng

p.quinquefolium

P.ginseng

P.notoginseng

P. quincuefolium

VMLRLLGEGLAESDDGNGAVERGRK VMLRLLGEGLABSDDGNGAVBRGRKW

P.ginseng

p.notoginseng

P. quinquefolium

IPSWGKTYLAVLSVYEWBGCNPLPPEENLFFSSPPFHEAKMWIYCRCTYUPMSYLYGKRYH

I PSWGKTYLAVL GVYENBGCNPLPPEENLFPSSPPFHPAKMWIYCRCTYMPMSYLYGKRY

P. ginseng

P. notoginseng

P. quinquefolium

LVQDLWWDGLHYFSBPFLKRNPFNKLRKR

LVQDLVWDGLHYRSBPELKRNPENKLRK

LVODLVWDGLHYFSEPELKRWPENKLRKR

p.ginseng

P. notoginseng

p.quinquefolium

CKBDLYYP:

280

280

280

\section{p.ginseng \\ P. notoginseng}

P. quínquefolium

P.ginseng

P. notoginseng

P. quingue folium

P.ginseng

P. notoginseng

P. quinquefolium

P.ginseng

P.notoginseng

P. quinquefolium

\section{p.ginseng}

P. notoginseng

P. quinquefolium

560

560

560

Figure 4 Alignment of DS amino acid residues from $P$. ginseng, $P$. quinquefolium, and $P$. notoginseng. $P$. ginseng DS and $P$. quinquefolius DS showing $99.5 \%$ identity, $P$. quinquefolius DS and $P$. notoginseng DS showing $99.0 \%$ identity, $P$. ginseng DS and $P$. notoginseng DS showing $98.7 \%$ identity. 
deposited in NCBI GenBank with Accession No. GU997679 and GU997680, respectively. All three DSs have 769 amino acid residues and display high similarity. At the protein level, DSs exhibit $99.5 \%$ identity between $P$. ginseng and $P$. quinquefolius, $99.0 \%$ identity between $P$. quinquefolius and $P$. notoginseng, and $98.7 \%$ identity between $P$. ginseng and $P$. notoginseng, indicating that $P$. ginseng and $P$. quinquefolius are more closely related. This conclusion agrees with that drawn from the alignment of $\operatorname{TrnK}$ and 18S rRNA sequences [42].

The oleanane-type saponin (Ro) has not been detected in $P$. notoginseng based on the phytochemical studies [2]. Surprisingly, two $P$. notoginseng singleton sequences (FW1NBNE02D50IR and FW1NBNE02D30JP) matched to $\beta$-AS of Panax ginseng. The presence of the $\beta$-ASs in $P$. notoginseng was further confirmed by RT-PCR and the PCR products were sequenced (Data not shown). The existence of the transcripts for $\beta$-ASs in P. notoginseng was seemingly conflicted with previous reports claiming that oleanane-type ginsenosides do not exist in $P$. notoginseng $[2,43]$. Therefore, we presumed that either oleanane-type ginsenosides were present in $P$. notoginseng at levels too low to be detected phytochemically, or oleanane-type ginsenosides in fact did not exist in $P$. notoginseng, despite the presence of $\beta-A S$, might due to the lack of biosynthetic genes downstream of $\beta$ AS.

\section{Discovery of the candidate CYP450s and UGTs might be involved in triterpene saponin biosynthesis by phylogenetic analysis}

Characterization of specific CYP450s or UGTs involved in triterpene saponin biosynthesis in Panax genus will facilitate to elucidation of the triterpene saponin biosynthetic pathway. CYP450s are generally involved in the biosynthesis of terpenoids, sterols, lignins, hormones, fatty acids, pigments, and phytoalexins in plants [44]. Some CYP450s are proposed to participate in the oxidation of the dammarane skeleton at C-12 and the other at C-6 toward the production of protopanaxadiol and protopanaxatriol, respectively $[10,45]$. Previous studies have characterized CYP88D6 from Glycyrrhiza uralensis (CYP85 clan) [23] and CYP93E1 from Glycine max (CYP71 clan) [46], both of which were involved in triterpene saponin biosynthesis. Therefore, the CYP450s belonging to CYP85 and CYP71 clan might be involved in ginsenoside biosynthesis in Panax genus. Glycosylation, catalyzed by glycosyltransferases (GTs), transfers the activated saccharides to an aglycone substrate in the modification on ginsenoside biosynthesis. This enzymatic conjugation based on glycosylation can stabilize the product and alter its physiological activity [47]. The dammarane- and oleanane-type aglycones have ginsenoside bioactivity after the glycosylation catalyzed by UGTs. UGT73K1 and UGT71G1 from Medicago truncatula [48] and UGT74M1 from Saponaria vaccaria [22] have been identified and characterized with functions in triterpene saponin biosynthesis. Recently, several CYP450s and UGTs were found as candidate genes involved in ginsenoside biosynthesis in $P$. quinquefolius and $P$. ginseng in our previous studies [15,29]. Particularly, one CYP450 (contig00248) and four UGTs (contig01001, contig14976, contig15451, and contig16321) were selected as candidate genes most likely to be involved in ginsenoside biosynthesis based on their MeJA-inducible and tissue specific expression patterns in P. quinquefolius [15].

In this study, a total of 174 CYP450 (Additional file 8) and 242 GT (Additional file 9) unique sequences were found in the $P$. notoginseng cDNA library. As reported for $P$. ginseng and other plants, enzymes in the same biosynthetic pathway are usually co-expressed $[14,48,49]$. Hence, $D S$ was expressed abundantly (with 1,018 reads) in the root of $P$. notoginseng, indicating that other genes in the triterpene saponin biosynthetic pathway might also expressed at higher levels in the $P$. notoginseng root. Based on this knowledge, CYP450- and UGT-unique sequences that contain more than 10 reads from the $P$. notoginseng root cDNA library were found as candidate enzymes involved in triterpene saponin biosynthesis. Thus, 25 CYP450s and 16 UGTs were selected, among which 15 CYP450s and 8 UGTs had full-length sequences after assembly and using RACE (rapid amplification of $\underline{\mathrm{c}} \mathrm{DNA}$ end) method. The primers used for RACE were listed in Additional file 10. These cDNA sequences have been submitted to the NCBI database and given accession number GU997664GU997678 for CYP450s and GU997656-GU997663 for UGTs.

The phylogenetic relationship between the15 fulllength CYP450s of $P$. notoginseng and characterized CYP450s from other plants was depicted in Figure 5. Four CYP450s (Pn00445, Pn00158, Pn01024, and Pn04451) belonged to the group representing the CYP85 clan which comprises CYP88D6 from G. uralensis, a licorice $\beta$-amyrin 11-oxidase with a key role in the biosynthesis of the triterpene sweetener glycyrrhizin [23] (Figure 5). Thus, the four CYP450s constituted a subgroup and were related to CYP88D6 (Figure 5). Interestingly, the transcript of Pn00158 had high identity to the P. quinquefolius candidate CYP450 (contig00248) [15] (Data not shown), which was likely to be involved in ginsenoside biosynthesis. Seven CYP450s (Pn02132, Pn02294, Pn01023, Pn13620, Pn01796, Pn03717, and Pn01705) belonged to the group representing the CYP71 clan (Figure 5). It is noteworthy that Pn02132 was phylogenetically close to CYP93E1 (Figure 5), which was the first triterpene hydroxylase identified from a plant species [46]. Therefore, Pn02132 and Pn00158 


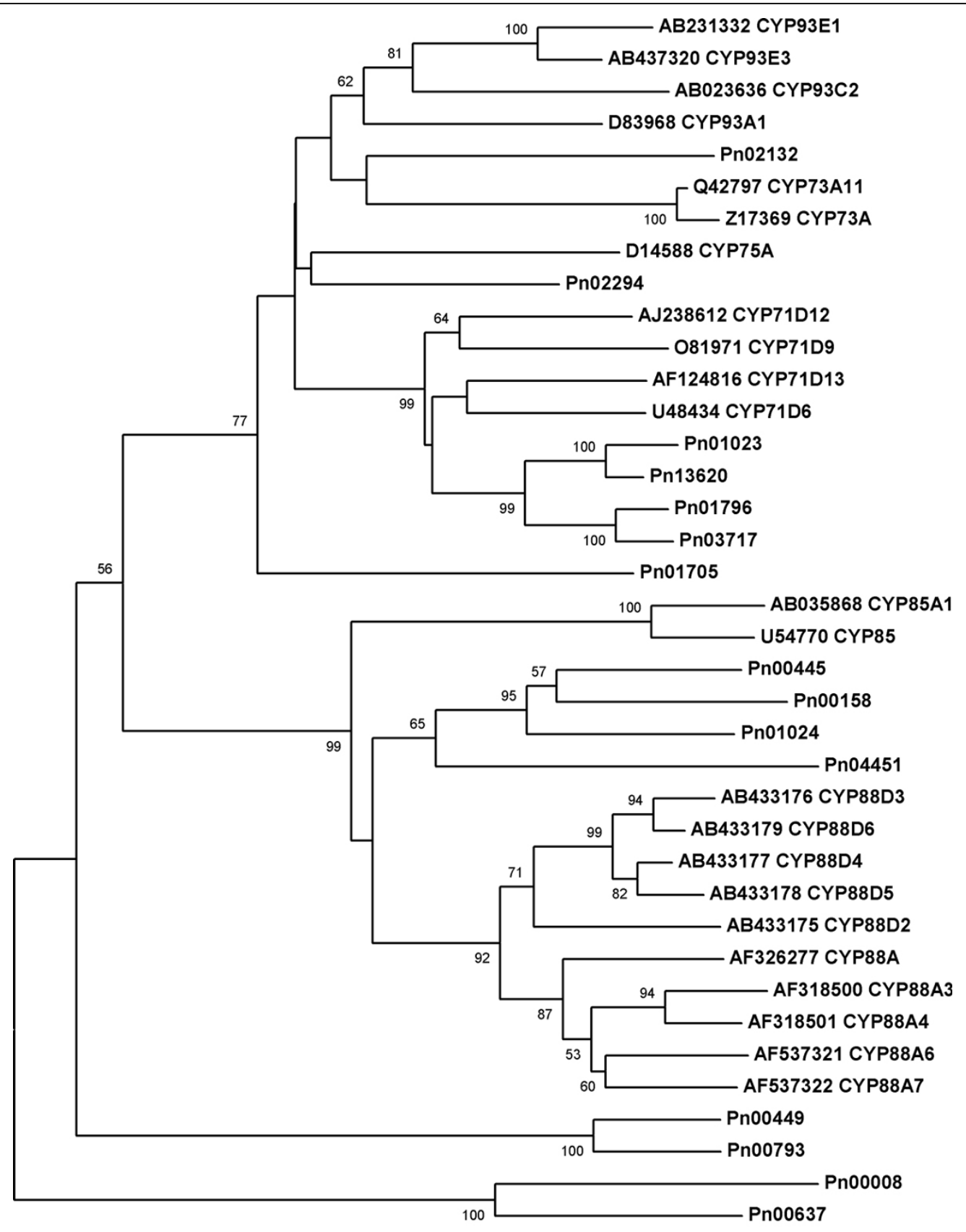

Figure 5 Phylogenetic analysis of full-length CYP450s from P. notoginseng and characterized CYP450s from other plants. Amino acid sequences were aligned using the CLUSTALW program, and evolutionary distances were computed using MEGA4 with the Poisson correction method. Bootstrap values obtained after 1000 replications are indicated on the branches. Values less than 50\% are not shown. The GenBank/ EMBL/DDBJ accession numbers of the sequences are: U48434 (Solanum chacoense), 081971 (Glycine max), AJ238612 (Catharanthus roseus), AF124816 (Mentha $\times$ piperita), Z17369 (Helianthus tuberosus), Q42797 (Glycine max), D14588 (Petunia x hybrida), AB035868 (Arabidopsis thaliana), U54770 (Solanum lycopersicum), AB433175 (Medicago truncatula), AB433176 (Medicago truncatula), AB433177 (Lotus japonicus), AB433178 (Lotus japonicus), AB433179 (Glycyrrhiza uralensis), AF537321 (Pisum sativum), AF537322 (Pisum sativum), AF318500 (Arabidopsis thaliana), AF318501 (Arabidopsis thaliana), AF326277 (Hordeum vulgare), AF135485 (Glycine max), AB231332 (Glycine max), AB023636 (Glycyrrhiza echinata), AB437320 (Glycyrrhiza uralensis), D83968 (Glycine max), X71657 (Solanum melongena), X71658 (Solanum melongena), X71656 (Solanum melongena), X71655 (Solanum melongena), L23209 (Zea mays), NM_001112599 (Zea mays), U29333 (Pisum sativum), U69134 (Arabidopsis thaliana), U18929 (Arabidopsis thaliana), AF150881 (Solanum lycopersicum $\times$ Solanum peruvianum), AF214008 (Brassica napus), U38416 (Arabidopsis thaliana), AF029856 (Sorghum bicolor), AF029858 (Sorghum bicolor), AJ583531 (Triticum aestivum). 
were lead candidate CYP450s involved in triterpene saponin biosynthesis.

Phylogenetic analysis using an unrooted distance tree showed the relationship of $P$. notoginseng UGT sequences to other functionally characterized members of plant UGT families (Figure 6). Three UGTs (Pn00082, Pn02086, and Pn13895) belonged to the group consisting of triterpene glycosyltransferases (UGT73K1, UGT71G1) from Medicago truncatula (Figure 6). Among them, Pn13895 was regarded as a lead candidate UGT responsible for triterpene saponin biosynthesis, because of its close relation to UGT71G1(Figure 6). Characterization of these candidate CYP450s and UGTs will pave the way to illustrate the biosynthetic pathways of triterpene saponins in $P$. notoginseng and other related Panax species. Although many of candidate genes involved in triterpene saponin biosynthesis were discovered in this study, the functional identification of these genes has not be carried out in this study. They will be the focus of study in the future

\section{Conclusions}

In this study, a large-scale 454-EST investigation of $P$. notoginseng root was performed based on 454 pyrosequencing. This 454-EST dataset from $P$. notoginseng root contribute significantly to provide a large number of transcripts for gene discovery in this medicinal plant. The description of the expressed genes and distribution of gene functions was illustrated according to GO analysis and KEGG assignment. A number of genes involved in triterpene saponin biosynthesis, including cytochrome P450s and glycosyltransferases, were discovered in our EST dataset. More importantly, a handful of candidate CYP450s and UGTs that are most likely to be involved in the biosynthesis of triterpene saponins were found based on phylogenetic analysis. Many transcription factors and EST-SSR markers were identified as well. These data will provide comprehensive information on gene discovery, transcriptome profiling, transcriptional regulation, and molecular markers for $P$. notoginseng. This study will contribute to further improvements on this medicinal plant through marker-assisted breeding or genetic engineering on this species as well as for other medicinal plants in the Araliaceae family.

\section{Methods}

Plant material

The 4-year-old Panax notoginseng cultivated on farms was routinely harvested for medical purposes. The $P$. notoginseng was collected from the fields of Wenshan County, Yunnan Province, China. After cleaning, the root tissues were cut into small pieces and immediately frozen in liquid nitrogen, and stored at $-80^{\circ} \mathrm{C}$ until further processing.

\section{RNA preparation}

Total RNA was isolated using the Plant RNA Isolation Mini Kit (BioTeke, Beijing, China). The total RNA was treated with DNase I (TURBO DNase; Ambion, USA) at a concentration of 1.5 units/ $\mu$ g of total RNA. The RNA quality was tested using $1 \%$ ethidium bromide-stained (EtBr-stained) agarose gels and the concentration was assessed using a GeneQuant100 spectrophotometer (GE Healthcare, UK) prior to cDNA synthesis.

\section{CDNA synthesis and 454 pyrosequencing}

The first-strand cDNA was produced using $2.1 \mu \mathrm{g}$ of total RNA extracted from the root of $P$. notoginseng according to the instructions provided with Clontech's SMART cDNA synthesis kit (Clontech, USA) with slight modifications as our previous study [15]: in order to remove the long poly $(\mathrm{A} / \mathrm{T})$ tails in cDNA sequences, a modified synthetic poly $(\mathrm{T})$ primer (5'-AAG CAG TGG TAT CAA CGC AGT GCA GT(20)VN-3') containing a $B s g I$ digestion site upstream of the poly $(\mathrm{T})$ segment was used in combination with the Clontech SMART IV primer to synthesize the first-strand cDNA. The cDNA was amplified using PCR Advantage II polymerase (Clontech, USA) to synthesize the double-strand cDNA (ds cDNA) with the following thermal profile: $1 \mathrm{~min}$ at $95^{\circ} \mathrm{C}$ followed by 19 cycles of $95^{\circ} \mathrm{C}$ for $15 \mathrm{sec}, 65^{\circ} \mathrm{C}$ for $30 \mathrm{sec}$, and $68^{\circ} \mathrm{C}$ for $6 \mathrm{~min}$. And then, $5 \mu \mathrm{l}$ of PCR product were electrophoresed in a $1 \%$ agarose gel to determine the amplification efficiency and quality. Approximately $13 \mu \mathrm{g}$ of amplified ds cDNA was purified using the PureLink TM PCR purification kit (Invitrogen, USA). Then the purified cDNA was treated with BsgI (NEB, USA) overnight at $37^{\circ} \mathrm{C}$ and recovered by QIAquick PCR Purification Kit (Qiagen, USA). Finally, a total of $10 \mu \mathrm{g}$ of ds cDNA was used for pyrosequencing with the GS FLX Titanium Kit.

\section{EST assembly and annotation}

The 454 raw read sequences were screened and trimmed for weak signals by GS FLX pyrosequencing software to yield high-quality (HQ) (> 99.5\% accuracy of single-base reads) sequences. The resulting HQ reads were then submitted to the Short Read Archive at NCBI and assigned the accession number SRX017444. The primer and adapter sequences were trimmed from the HQ sequences, followed by removing the sequences shorter than $50 \mathrm{bp}$ from the clean ESTs before assembly. Then, the data from the 454 read sequences were assembled into unique sequences (including contigs and singletons) using 454 GS De Novo Assembler software v2.0.01.14 (454 Life Sciences, Roche) with a quality score threshold set at 40 .

The assembled unique sequences were first searched for sequence similarities against the NCBI non- 


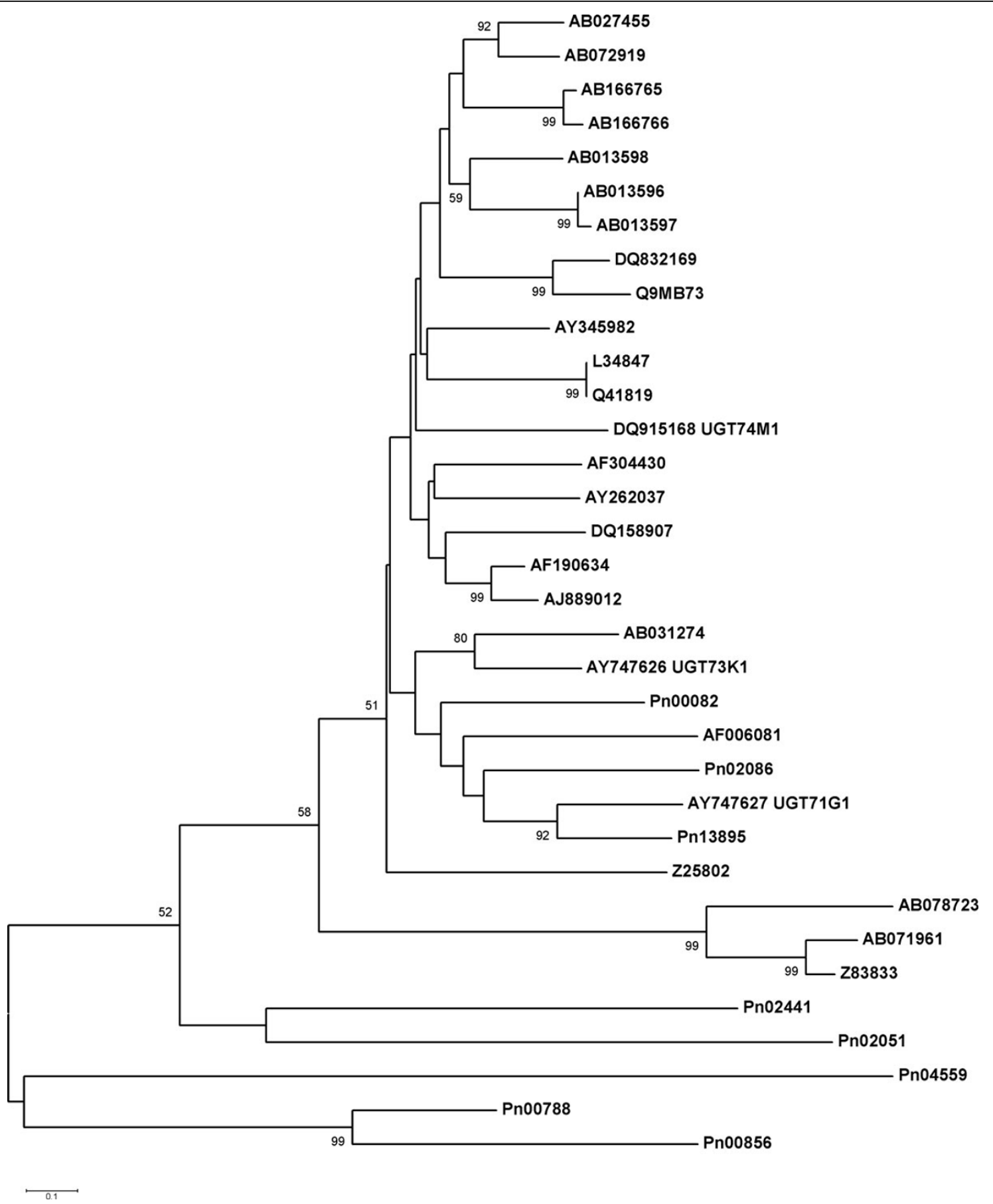

Figure 6 Phylogenetic analysis between full-length UGTs of $P$. notoginseng and characterized UGTs from other plants. Amino acid sequences were aligned using the program CLUSTALW and evolutionary distances were computed using the Poisson correction method with MEGA4. Bootstrap values obtained after 1000 replications are indicated on the branches. Values less than 50\% are not shown. The GenBank/ EMBL/DDBJ accession numbers of the sequences are AY747627 (Medicago truncatula), AY747626 (Medicago truncatula), DQ915168 (Vaccaria hispanica), AB013598 (Glandularia × hybrida), AB027455 (Petunia $\times$ hybrida), AB013597 (Perilla frutescens var. crispa), AB013596 (Perilla frutescens var. crispa), AY262037 (Crocus sativus), AY345982 (Stevia rebaudiana), Q41819 (Zea mays), DQ158907 (Arabidopsis thaliana), Q9MB73 (Citrus unshiu), AB166766 (Eucalyptus perriniana), AB166765 (Eucalyptus perriniana), AJ889012 (Solanum lycopersicum), AB072919 (Nicotiana tabacum), DQ832169 (Vitis labrusca), AF190634 (Nicotiana tabacum), AF304430 (Brassica napus), AB078723 (Aspergillus oryzae), Z83833 (Arabidopsis thaliana), AB071961 (Panax ginseng), AB031274 (Scutellaria baicalensis), Z25802 (Petunia $\times$ hybrida), L34847 (Zea mays). 
redundant nucleotide $(\mathrm{Nt})$ database using the BLASTN algorithm with $E$-value cut-off of $10^{-5}$ to find and remove ribosomal RNA sequences [50]. And then, the remaining sequences were searched against the public databases including the Arabidopsis protein database at The Arabidopsis Information Resource (TAIR; http:// www.arabidopsis.org) (version Tair9), SwissProt protein database (http://www.expasy.ch/sprot; released on 06/ $19 / 2009)$, and the NCBI non-redundant protein $(\mathrm{Nr})$ database (http://www.ncbi.nlm.nih.gov; released on 06/ 23/2009) using the BLASTX algorithm with an E-value cut-off of $10^{-5}$. The functional categories of these unique sequences were further analyzed using the Gene Ontology (GO) database. The unique sequences were categorized according to GO terms based on AGI codes and TAIR GO slim provided by TAIR.

\section{Pathway assignment with KEGG database}

Pathway assignments were carried out according to the Kyoto Encyclopedia of Genes and Genome (KEGG) mapping (http://www.genome.ad.jp/kegg/kegg2.html) (version KEGG 50) [33]. Enzyme commission (EC) numbers were assigned to unique sequences after BLASTX searches with an $E$-value cut-off of $10^{-5}$ upon against the KEGG database. The unique sequences were assigned to special biochemical pathways according to the corresponding EC distribution in the KEGG database.

\section{Simple sequence repeat (SSR) detection}

The total unique sequences were searched to determine the composition, frequency, and distribution of simple sequence repeats (SSRs) using an online SSR identification tool - SSRIT (Simple Sequence Repeat Identification Tool) (http://www.gramene.org/db/markers/ssrtool) [51]. The search parameters for the maximum motiflength group were set to recognize hexamers and the minimum number of repeats was set to five.

\section{Screening of CYP450 or UGT unique sequences encoding} enzymes responsible for triterpene saponin biosynthesis

The DS transcript was much more abundant in $P$. notoginseng (1,018 reads), suggesting the other genes encoding enzymes in the same biosynthetic pathway were also expressed at much higher levels in the former species. For CYP450s and UGTs, each isozyme with more than ten reads in $P$. notoginseng is arbitrarily considered a candidate involved in the biosynthesis of triterpene saponins. The screening of CYP450s and UGTs was performed according to phylogenetic analysis.

\section{Production of full-length cDNA sequences for CYP450s and UGTs using RACE technology}

Primers listed in Additional file 10 were synthesized according to selected CYP450s and UGTs EST sequences. The 5' or 3' ends of cDNAs were amplified using a SMART RACE cDNA amplification kit (Clontech, USA) from total RNA of $P$. notoginseng root and cloned into $\mathrm{T}$ easy Vector (Promega, USA) for Sanger sequencing. Then the full-length cDNA of each gene was generated by assembly of the corresponding EST sequence and 5' and/or 3'end sequences.

\section{Phylogenetic analysis}

Distances between each clone were calculated with the CLUSTAL W program [52]. The indicated scale represents 0.1 amino acid substitutions per site. Amino acid sequences were aligned using the CLUSTAL W program and evolutionary distances were computed using the Poisson correction method, and a Neighbor-Joining (NJ) tree was constructed with MEGA4. Bootstrap values obtained after 1000 replications are given on the branches. Values less than $50 \%$ are not shown.

\section{Additional material}

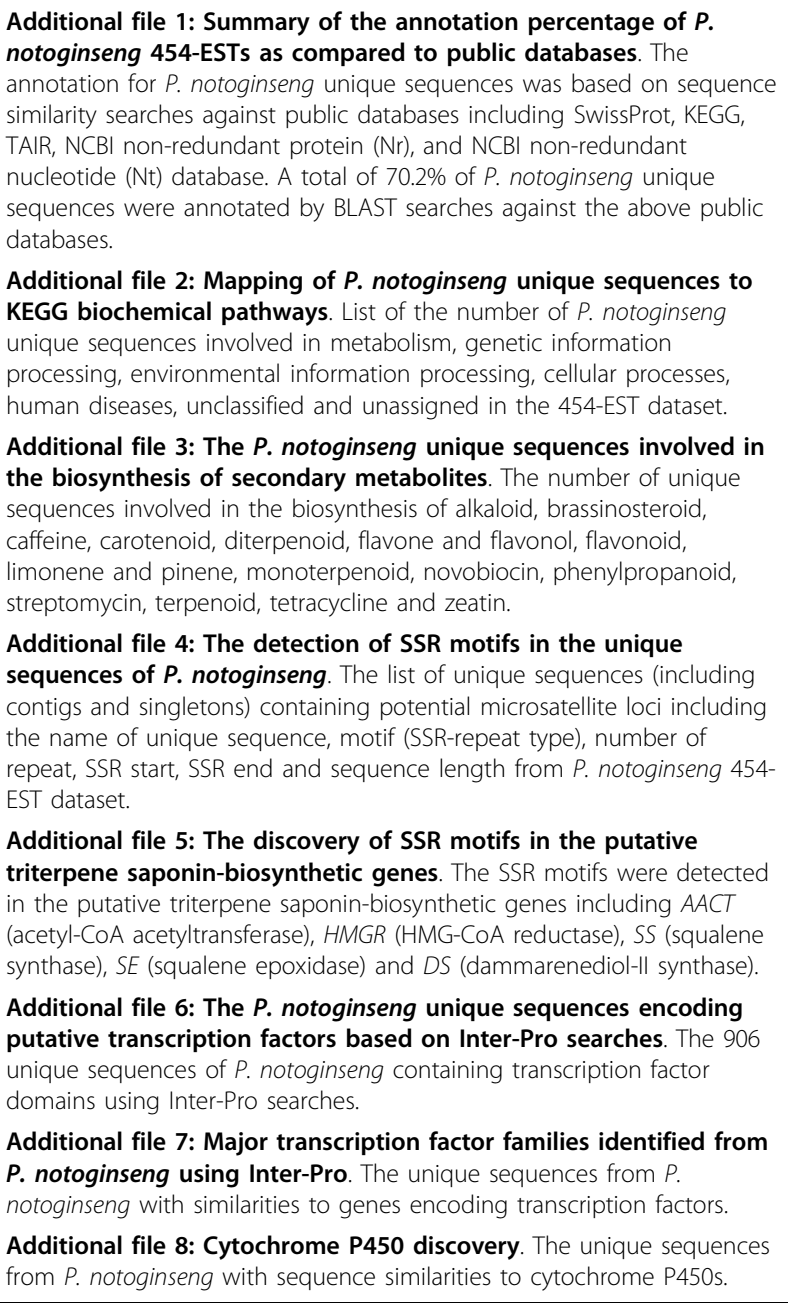


Additional file 9: Glycosyltransferase discovery. The unique sequences from $P$. notoginseng with sequence similarities to glycosyltransferase.

Additional file 10: The primers used for RACE in this study. The primers used in $5^{\prime}$-RACE for the amplification of unique sequences including Pn01024, Pn02132, Pn03717, and Pn00788.

\section{List of abbreviations}

$\beta$-AS: $\beta$-amyrin synthase; BLAST: basic local alignment search tool; bp: base pair; cDNA: complementary DNA; CYP450: cytochrome P450; DS: dammarenediol-II synthase; EST: expressed sequence tag; GGDP: geranylgeranyl diphosphate; GO: gene ontology; GT: glucosyltransferase; KEGG: Kyoto encyclopedia of genes and genomes; MeJA: methyl jasmonate; NCBI: National Center for Biotechnology Information; NGS: next-generation sequencing; RACE: rapid amplification of CDNA end; SSR: simple sequence repeat; TAIR: the Arabidopsis Information Resource; UGT: UDPglucosyltransferase.

\section{Acknowledgements}

This work was supported by the National Natural Science Foundation of China (grant No. 81130069 and 30900113), and the preferred foundation of Ministry of human resources and social security of the People's Republic of China (2009-1011). The authors thank Dr. Chang Liu for his revision on this manuscript and Dr. Xi-Wen Li for his assistance with sample collection.

\section{Author details}

${ }^{1}$ The Key Laboratory of Bioactive Substances and Resources Utilization of Chinese Herbal Medicine, Ministry of Education, Institute of Medicinal Plant Development, Chinese Academy of Medical Sciences \& Peking Union Medical College, Beijing 100193, P. R. China. ${ }^{2}$ School of Pharmacy, Guilin Medical University, Guilin 541004, China. ${ }^{3}$ China National Center for Biotechnology Development, Beijing 100036, China. ${ }^{4}$ Shanghai University of Traditional Chinese Medicine, Shanghai, China. ${ }^{5}$ Guanzhou Pharmaceutical Holding Limited, Guangzhou, China. ${ }^{6}$ Centre de Recherche Public-Santé, Luxembourg, L-1526 Luxembourg, Belgium.

\section{Authors' contributions}

HML contributed to the CDNA sample preparation, data analysis and manuscript writing. CS and YZS participated in the design of the study, the tissue sample collection, and the data analysis. QW helped with RNA extraction, gene cloning and the generation of the phylogenetic tree for CYP450s and UGTs. YL contributed to the bioinformatic analysis and helped with the RNA extraction. YYN helped with RNA extraction and CDNA amplification. JYS, XLC, HXX, CYL, JYL and AS participated in the study design and discussed the results. This work was conducted in the laboratory of SLC, who initiated the 454-sequencing projects and contributed to the evaluation and discussion of the results as well as to the revision of the manuscript. All authors have read and approved the final manuscript.

\section{Competing interests}

The authors declare that they have no competing interests.

Published: 23 December 2011

\section{References}

1. Briskin DP: Medicinal plants and phytomedicines. Linking plant biochemistry and physiology to human health. Plant Physiol 2000, 124(2):507-514.

2. Wang CZ, McEntee E, Wicks S, Wu JA, Yuan CS: Phytochemical and analytical studies of Panax notoginseng (Burk.) F.H.Chen. J Nat Med 2006, 60:97-106.

3. Ng TB: Pharmacological activity of sanchi ginseng (Panax notoginseng). J Pharm Pharmacol 2006, 58(8):1007-1019.

4. Hong DY, Lau AJ, Yeo CL, Liu XK, Yang CR, Koh HL, Hong Y: Genetic diversity and variation of saponin contents in Panax notoginseng roots from a single farm. J Agr Food Chem 2005, 53(22):8460-8467.
5. Wu W, Zhang XM, Liu PM, Li JM, Wang JF: Effects of Panax notoginseng saponin $\mathrm{Rg} 1$ on cardiac electrophysiological properties and ventricular fibrillation threshold in dogs. Acta Pharmacol Sin 1995, 16(5):459-463.

6. Chen JC, Chen LD, Tsauer W, Tsai CC, Chen BC, Chen YJ: Effects of Ginsenoside Rb2 and Rc on inferior human sperm motility in vitro. Am J Chinese Med 2001, 29(1):155-160.

7. Li W, Fitzloff JF: A validated method for quantitative determination of saponins in notoginseng (Panax notoginseng) using high-performance liquid chromatography with evaporative light-scattering detection. $J$ Pharm Pharmacol 2001, 53(12):1637-1643.

8. Gan FY, Zhen GZ: Chemical composition studies of Panax notoginseng. Chin Pharm J 1992, 27:138-143.

9. Haralampidis K, Trojanowska M, Osbourn AE: Biosynthesis of triterpenoid saponins in plants. Adv Biochem Eng/Biotechnol 2002, 75:31-49.

10. Tansakul P, Shibuya M, Kushiro T, Ebizuka Y: Dammarenediol-II synthase, the first dedicated enzyme for ginsenoside biosynthesis, in Panax ginseng. FEBS Lett 2006, 580(22):5143-5149.

11. Kushiro T, Shibuya M, Ebizuka Y: Beta-amyrin synthase-cloning of oxidosqualene cyclase that catalyzes the formation of the most popular triterpene among higher plants. Eur J Biochem 1998, 256(1):238-244.

12. Herrera JBR, Bartel B, Wilson WK, SPT M: Cloning and characterization of the Arabidopsis thaliana lupeol synthase gene. Phytochemistry 1998, 7:1905-1911.

13. Corey EJ, Matsuda SP, Bartel B: Isolation of an Arabidopsis thaliana gene encoding cycloartenol synthase by functional expression in a yeast mutant lacking lanosterol synthase by the use of a chromatographic screen. Proc Natl Acad Sci USA 1993, 90(24):11628-11632.

14. Han JY, Kwon YS, Yang DC, Jung YR, Choi YE: Expression and RNA interference-induced silencing of the dammarenediol synthase gene in Panax ginseng. Plant Cell Physiol 2006, 47(12):1653-1662.

15. Sun C, Li Y, Wu Q, Luo H, Sun Y, Song J, Lui EM, Chen S: De novo sequencing and analysis of the American ginseng root transcriptome using a GS FLX Titanium platform to discover putative genes involved in ginsenoside biosynthesis. BMC Genomics 2010, 11:262.

16. Parkinson J, Blaxter M: Expressed sequence tags: an overview. Methods Mol Biol 2009, 533:1-12.

17. Parkinson J: Expressed sequence tags (ESTS) generation and analysis Humana Press; 2009.

18. Rudd S: Expressed sequence tags: alternative or complement to whole genome sequences? Trends Plant Sci 2003, 8(7):321-329.

19. Fields C: Analysis of gene expression by tissue and developmental stage. Curr Opin Biotechnol 1994, 5:595-598.

20. Murataa J, Bienzleb D, Brandlec JE, Sensend CW, Luca VD: Expressed sequence tags from Madagascar periwinkle (Catharanthus roseus). FEBS Lett 2006, 580:4501-4507.

21. Li Y, Luo H, Sun C, Song J, Sun Y, Wu Q, Wang N, Yao H, Steinmetz A, Chen S: EST analysis reveals putative genes involved in glycyrrhizin biosynthesis. BMC Genomics 2010, 11:268.

22. Meesapyodsuk D, Balsevich J, Reed DW, Covello PS: Saponin biosynthesis in Saponaria vaccaria. CDNAs encoding beta-amyrin synthase and a triterpene carboxylic acid glucosyltransferase. Plant Physiol 2007, 143(2):959-969.

23. Seki H, Ohyama K, Sawai S, Mizutani M, Ohnishi T, Sudo H, Akashi T, Aoki T, Saito K, Muranaka T: Licorice beta-amyrin 11-oxidase, a cytochrome P450 with a key role in the biosynthesis of the triterpene sweetener glycyrrhizin. Proc Natl Acad Sci USA 2008, 105(37):14204-14209.

24. Varshney RK, Graner A, Sorrells ME: Genic microsatellite markers in plants: features and applications. Trends Biotechnol 2005, 23(1):48-55.

25. Morozova O, Hirst M, Marra MA: Applications of new sequencing technologies for transcriptome analysis. Annu Rev Genom Hum Genet 2009, 10(1):135-151.

26. Simon SA, Zhai J, Nandety RS, McCormick KP, Zeng J, Mejia D, Meyers BC: Short-read sequencing technologies for transcriptional analyses. Annu Rev Plant Biol 2009, 60(1):305-333

27. Luo H, Li Y, Sun C, Wu Q, Song J, Sun Y, Steinmetz A, Chen S: Comparison of 454-ESTs from Huperzia serrata and Phlegmariurus carinatus reveals putative genes involved in lycopodium alkaloid biosynthesis and developmental regulation. BMC Plant Biol 2010, 10:209.

28. Pop M, Salzberg SL: Bioinformatics challenges of new sequencing technology. Trends Genet 2008, 24(3):142-149. 
29. Chen S, Luo H, Li Y, Sun Y, Wu Q, Niu Y, Song J, Lv A, Zhu Y, Sun C, Steinmetz A, Qian Z: 454 EST analysis detects genes putatively involved in ginsenoside biosynthesis in Panax ginseng. Plant Cell Rep 2011, 30:1593-1601.

30. Audic S, Claverie JM: The significance of digital gene expression profiles. Genome Res 1997, 7(10):986-995.

31. Wang W, Vinocur B, Shoseyov O, Altman A: Role of plant heat-shock proteins and molecular chaperones in the abiotic stress response. Trends Plant Sci 2004, 9(5):244-252.

32. Berardini TZ, Mundodi S, Reiser L, Huala E, Garcia-Hernandez M, Zhang P, Mueller LA, Yoon J, Doyle A, Lander G, et al: Functional annotation of the Arabidopsis genome using controlled vocabularies. Plant Physiol 2004, 135(2):745-755.

33. Kanehisa M, Goto S: KEGG: Kyoto Encyclopedia of Genes and Genomes. Nucleic Acids Res 2000, 28(1):27-30.

34. Sharma RK, Bhardwaj P, Negi R, Mohapatra T, Ahuja PS: Identification, characterization and utilization of unigene derived microsatellite markers in tea (Camellia sinensis L.). BMC Plant Biol 2009, 9:53.

35. Kumpatla SP, Mukhopadhyay S: Mining and survey of simple sequence repeats in expressed sequence tags of dicotyledonous species. Genome 2005, 48:985-998.

36. Senthilvel S, Jayashree B, Mahalakshmi V, Kumar PS, Nakka S, Nepolean T, Hash C: Development and mapping of simple sequence repeat markers for pearl millet from data mining of expressed sequence tags. BMC Plant Biol 2008, 8:119.

37. Varshney Rk, Thiel T, Stein N, Langridge P, Graner A: In silico analysis on frequency and distribution of microsatellites in ESTs of some cereal species. Cell Mol Biol Lett 2002, 7:537-546.

38. Wu Q, Song J, Sun Y, Suo F, Li C, Luo H, Liu Y, Li Y, Zhang X, Yao H, et al: Transcript profiles of Panax quinquefolius from flower, leaf and root bring new insights into genes related to ginsenosides biosynthesis and transcriptional regulation. Physiol Plant 2010, 138(2):134-149.

39. Singh K, Foley RC, Onate-Sanchez L: Transcription factors in plant defense and stress responses. Curr Opin Plant Biol 2002, 5(5):430-436.

40. Yanhui C, Xiaoyuan $Y$, Kun $H$, Meihua L, Jigang L, Zhaofeng G, Zhiqiang $L$, Yunfei Z, Xiaoxiao W, Xiaoming Q, et al: The MYB transcription factor superfamily of Arabidopsis: expression analysis and phylogenetic comparison with the rice MYB family. Plant Mol Biol 2006, 60(1):107-124.

41. Green KA, Prigge MJ, Katzman RB, Clark SE: CORONA, a member of the class III homeodomain leucine zipper gene family in Arabidopsis, regulates stem cell specification and organogenesis. Plant Cell 2005, 17(3):691-704.

42. Zhu S, Fushimi H, Cai S, Komatsu K: Phylogenetic relationship in the genus Panax: inferred from chloroplast trnK gene and nuclear 18S rRNA gene sequences. Planta Med 2003, 69(7):647-653.

43. Dong TT, Cui XM, Song ZH, Zhao KJ, Ji ZN, Lo CK, Tsim KW: Chemical assessment of roots of Panax notoginseng in China: regional and seasonal variations in its active constituents. J Agr Food Chem 2003, 51(16):4617-4623.

44. Meijer AH, Souer E, Verpoorte R, Hoge JHC: Isolation of cytochrome P450 CDNA clones from the higher plant Catharanthus roseus by a PCR strategy. Plant Mol Biol 1993, 22:379-383.

45. Jung JD, Park HW, Hahn Y, Hur CG, In DS, Chung HJ, Liu JR, Choi DW: Discovery of genes for ginsenoside biosynthesis by analysis of ginseng expressed sequence tags. Plant Cell Rep 2003, 22(3):224-230.

46. Shibuya M, Hoshino M, Katsube $Y$, Hayashi H, Kushiro T, Ebizuka Y: Identification of beta-amyrin and sophoradiol 24-hydroxylase by expressed sequence tag mining and functional expression assay. FEBS $J$ 2006, 273(5):948-959.

47. Hefner T, Arend J, Warzecha H, Siems K, Stockigt J: Arbutin synthase, a novel member of the NRD1beta glycosyltransferase family, is a unique multifunctional enzyme converting various natural products and xenobioticsy. Bioorgan Med Chem 2002, 10:1731-1741.

48. Achnine L, Huhman DV, Farag MA, Sumner LW, Blount JW, Dixon RA: Genomics-based selection and functional characterization of triterpene glycosyltransferases from the model legume Medicago truncatula. Plant $J$ 2005, 41(6):875-887.

49. Lee MH, Jeong JH, Seo JW, Shin CG, Kim YS, In JG, Yang DC, Yi JS, Choi YE: Enhanced triterpene and phytosterol biosynthesis in Panax ginseng overexpressing squalene synthase gene. Plant Cell Physiol 2004, 45(8):976-984.
50. Altschul SF, Gish W, Miller W, Myers EW, Lipman DJ: Basic local alignment search tool. J Mol Biol 1990, 215(3):403-410.

51. Temnykh S, DeClerck G, Lukashova A, Lipovich L, Cartinhour S, McCouch S: Computational and experimental analysis of microsatellites in Rice (Oryza sativa L.): frequency, lengthVariation, transposon associations, and genetic marker potential. Genome Res 2001, 11:1441-1452.

52. Thompson JD, Higgins DG, Gibson TJ: CLUSTAL W: improving the sensitivity of progressive multiple sequence alignment through sequence weighting, position-specific gap penalties and weight matrix choice. Nucleic Acids Res 1994, 22(22):4673-4680.

doi:10.1186/1471-2164-12-S5-S5

Cite this article as: Luo et al:: Analysis of the transcriptome of Panax notoginseng root uncovers putative triterpene saponin-biosynthetic genes and genetic markers. BMC Genomics 2011 12(Suppl 5):S5.

\section{Submit your next manuscript to BioMed Central and take full advantage of:}

- Convenient online submission

- Thorough peer review

- No space constraints or color figure charges

- Immediate publication on acceptance

- Inclusion in PubMed, CAS, Scopus and Google Scholar

- Research which is freely available for redistribution

Submit your manuscript at www.biomedcentral.com/submit
C Biomed Central 